\title{
Save Odysseus: An Escape Room as a Content Gamification Activity for Enhancing Collaboration and Resilience in the School Context
}

\author{
Evangelia E. Moula \\ Hellenic Open University, Patras, Greece \\ Konstantinos D. Malafantis \\ National and Kapodistrian University, Athens, Greece
}

\begin{abstract}
This paper describes the design and the implementation of an educational escape room with an Odyssey theme, in accordance with the content gamification model. Escape rooms are part of the gamification strategies adopted in education, so that students get actively engaged and at the same time achieve cognitive or pedagogical goals effortlessly. The specific escape room was developed as a playful alternative to the standard revision stage of the Odyssey, but also included interdisciplinary elements (Geography and Mathematics). The research methodology was exploratory, as a minimum foundation for further research, and quantitative. Escape room's main goal was to fight students' boredom and engage them in an entertaining learning process. The main research question was: do the vulnerable students (with low school performance) profit from this procedure and cultivate a positive attitude towards learning?
\end{abstract}

According to the results, the escape room enhanced engagement, collaboration, communication, and creativity, and mitigated stress through collaboration, which subsequently fosters resilience in the school context. It was also welcomed as beneficial pedagogical activity and caused, to an overwhelming extent, the enthusiasm of the participants. The (male) gender and the earlier experience of an escape room contributed to the more positive reception of the activity. Lowperformance students expressed their satisfaction and fervent approval of the experience and outscored those of high performance in almost all variables measured, proving the pedagogical value of the escape room's use.

Keywords: Escape room, education, gamification, collaboration, resilience, the Odyssey

\section{Introduction}

Taking into account the low socio-financial background of our school's population, the high school dropout rate and the low-to-mediocre grades of the previous school year in the subject of Odyssey, -a fact that reveals reduced interest of the students in it-, we decided to explore the possibilities of an escape room for cultivating resilience skills in our vulnerable students -as a presupposition to their positive attitude towards learning- through a playful process. More specifically, we implemented an escape room with an Odyssey theme, to fight students' 
boredom, provide motivation, engage them in an entertaining learning process, stimulate teamwork, boost collaboration and communication and consequently reinforce their positive attitude towards learning, as an integral and indispensable constituent element of school resilience.

The importance of the research lies in the fact that there are no similar researches about the use of escape rooms in theoretical fields, such as the study of ancient Greek literature, which is attempted here. Through this escape room we aimed both at achieving cognitive goals, as well as at enhancing positive disposition towards school and learning. Its contribution to conquering the cognitive goals is not examined in this paper. The research methodology was exploratory, as a minimum foundation for further research, and quantitative, applied through a questionnaire to asses various factors of the students' experience in a measurable way. The questions were formulated, so as to cover the main research questions.

Main research questions were:

- Does an escape room improve students' collaboration?

- Does it stimulate students' active engagement?

- Does collaboration mitigate anxiety of performance and enhance school resilience?

- Do students enjoy such an educational experience and express desire to repeat it?

- Do students evaluate it as pedagogically significant?

- Does students' satisfaction with the escape room depend on their school performance, previous similar experience or gender?

- Do the vulnerable students (with low school performance) profit from this procedure and cultivate a positive attitude towards learning?

- Secondary questions dealt with the measurement of the escape room's quality in terms of the level of puzzles' difficulty and timing. Questions evaluating the cognitive aspects are omitted here.

In the beginning, students were not eager to participate, expressing their reservation towards typical educational practices. These minor difficulties in the implementation were attributed to the students' predetermined, restrictive interpretative framing and their prejudiced stance toward all kinds of educational activities, but they were successfully overcome.

\section{Theoretical frame}

\subsection{Collaboration and resilience in school context}

The dominant goal of education in today's Learning Society is, in addition to teaching useful and necessary knowledge, the development and cultivation of soft skills (21st Century Skills), which will allow each learner to become an independent thinker and active citizen of the 21st century (Binkley at al., 2012; Caldwell \& Longmuir, 2010). Key or core competencies (Voogt \&. Roblin, 2010) which are common in all the above frameworks are the 4 C.: Collaboration, Communication, Creativity, Critical thinking.

The value of collaborative learning has long been supported and demonstrated, from Piaget's social constructivism and Vygotsky's sociocultural theory of learning to the theory of collective intelligence by Jenkins et.al (2009). In our time it is gaining a more and more prominent role in the proposed skills development models (Wagner, 2010).

Kuhn (2015) argues that collaboration as a process leads to a desirable individual or group outcomes, such as successful problem solving and enhanced intellectual development. Recent research focuses on assessing the impact of team size and communication on achieving specific goals. 
Collaboration and communication have been also shown to promote knowledge building, development of complex thinking (expression, formulation, argumentation, and negotiation of meanings), in-depth processing of information through group formation and active participation of learners in it.

Participation stimulates self-esteem and enhances the positive attitude towards the school environment, strengthening the resilience of learners. So two-way feedback is activated, where collaboration works in favor of resilience and vice versa. It is considered (Bernard, 1991) that children who have developed mental resilience have developed skills such as critical thinking, initiative and the ability to solve problems.

Nevertheless, although some children have an inherent predisposition to develop mental resilience, most of the above characteristics are a product of the learning process (Higgins, 1994). The prevailing view in the school environment today is that instead of focusing on eliminating problem behaviors, we should aim for prosperity and the promotion of positive behaviors (Nicotera \&. Laser-Maira, 2016). Features that contribute to this are connection, trust, and contribution (Lerner et al., 2005) which in turn are cultivated through collaboration.

Taking all the above into account, in this project, we focused in the first place on collaboration and communication through the participation in the escape room, not only as catalysts for cognitive development but mainly as propellers of psychological resilience in the school environment.

\subsection{Play, Game and learning}

The example of the adoption of play and gaming strategies to simulate real-life conditions, to disseminate knowledge, and to support moral and social development, has a very long history. Play is inherent in human nature and in all living creatures and "is more ancient than culture"(Huizinga, 1994) It has always been a natural daily behavior, especially for children, emanating from their inner selves. Dempsey, Haynes, Lucassen, and Casey (2002) attempting a more detailed definition, states that "a game is a set of activities involving one or more players It has goals, constraints, payoffs, and consequences. A game is a rule-guided and artificial in some respects. Finally, a game involves some aspect of competition, even if that competition is with oneself". It is a step outside of "real" life in a temporary sphere of activity on its own. Every child knows very well that it is just pretending, or that it is only participating for fun [14].

Prensky (2001) arguing for the need to change the methodological approach to learning and integrate a playful strategy, compares young people's ability to memorize Pokemon's characters and evolutions with their indifference and lack of interest to do the same with geographical details, concluding that it's knowledge presentation that key-factor that educators should take into account to achieve better learning results.

However, edutainment, the combination of entertainment and education, of which educational games are a part, has also been demoted as "chocolate-coated broccoli" (Bruckman, 1999) and external relationship with learning. The use of games in education provokes a variety of reactions (Moula, 2012), ranging from fervent enthusiasm (Mitchell \& Saville-Smith, 2004) to extreme skepticism (Anderson \& Dill, 2000) with a wide range of arguments and on both sides.

\subsubsection{Game-based learning: Digital Game-based learning (DGBL)}

Teachers have used game-based elements for decades. Reward-based and meaningful learning have been explored in detail. Game-based learning does not replace traditional learning but is considered as an alternative medium. The trend for GBL began in 2001, when Prensky 
introduced the concept of game-based digital learning (GBL) and which in turn profoundly affected the way the academic world developed the GBL standards and requirements in educational settings. The supremacy of computer games as the dominating paradigm in the field is unquestionable.

Game-based learning refers to the innovative learning approach derived from the use of computer games that possess educational value or other kinds of software applications that use games for learning and education purposes (Tang \& Hanneghan, 2011). The intent of gamebased learning is to bridge the gap between the in and out of school everyday culture of digital natives, making learning more relevant and appealing to motivate learners to acquire knowledge and skills, transferable into reality.

They can also provide ways to improve students' learning experience and outcomes and can efficiently be applied for engaging school-age students into teaching subjects that are considered 'difficult', strenuous and/or boring as well (Papadakis \& Kalogiannakis, 2019).

Various research initiatives, such as the Games-To-Teach project in the United States (Squire \& Jenkins, 2004) occasionally attempt to explore the potential of videogames to support learning. This trend is mainly based on the logic of taking advantage of the participatory culture of young people (Steinkuhler et al., 2012).

It is however argued (Bogost, 2011) that e-learning advocates make a crucial misunderstanding. Game developers and gamers criticize the GBL on the grounds that it incorrectly evaluates games, considering their secondary properties such as grades and levels as their main characteristics, though the most important one is games' interaction with behavioral complexity. Finally, it is even argued that games should be approached in education only in the context of media arts and should be considered as a special case of popular art, and as another example which, due to its exceptional multimodal nature, crosses the imaginary boundaries in the artistic practice of education, incorporating narration, dramatic action, music, visual design, and media techniques. Much recent research, however, suggests that as a student's performance in the game improves, so does his or her decision-making skills and overall positive attitudes and feelings that contribute to learning (Squire, 2006).

However, there is little evidence of how games are used and how well they are successfully incorporated into teaching practices. There is no sufficient data on which teaching subjects games are most successful in producing a desired or intended result, nor on how they are used by teachers (McClarty et al., 2012).

\subsubsection{Non-digital game-based learning models: Gamification}

While an overwhelming percentage of GBL research refers to the use of digital games and technology, it has been recognized by the academic community that GBL can also mean the adoption of non-digital models (Baker, Navarro \& Van Der Hoek, 2005) Nevertheless, research on the design, development, and evaluation of non-digital applications lags behind the prevailing research of their digital counterparts.

Even if game-based learning and gamification have overlapping research literature, shared design toolboxes [28] and the same emphasis on added learning value beyond entertainment, they are of a different nature. While game-based learning approaches involve the design of complete games (Deterding, Dixon, Khaled \& Nacke 2011), gamification approaches focus on changing an existing learning process so that users perceive it as a game (Landers, Auer, Collmus \& Armstrong, 2018). 
The awareness of the need to devise new teaching methods that attract demanding students, except for the use of digital games, further led to the adoption of gamification as a learning enhancement mechanism. The term Gamification has been defined as "using game-based mechanics, aesthetics and game thinking to engage people, motivate action, promote learning, and solve problems" (Kapp, 2012: 10) "to make activities more engaging and fun" (Kim, 2011) "in non-game contexts" (Deterding et al., 2011) with the aim of changing user behavior as well as increasing participation and commitment. The term first appeared in 2008 in the digital media industry and did not gain widespread recognition until 2012, when Kapp published The Gamification of Learning and Instruction, a milestone in the corresponding literature. While the term is relatively new, there seems to be general agreement about its basic tenants. Many of the gamification elements are nothing but new names applied to given concepts, such as game objectives in place of course requirements, quests instead of assignments, points instead of grades and so on (Bucket \& Nicholson, 2016: 66).

Gamification encourages creative thinking, does not stigmatize failure, and provides the player with a sense of control over the process. It is not just badges and rewards (Kapp, 2012) but instead a sense of involvement, of accomplishment and of tackling a challenge.

There are two types of gamification. In content gamification, the content is altered to be more game-like by using elements such as challenges, feedback loops, and storytelling. It enhances engagement while foregrounding the content. Adding these elements makes the content more game-like but doesn't turn the content into a game. It simply provides contextual or embedded activities to the content being taught.

In structural gamification game elements are added to the structure of the content, which remains unaltered. The aim is to encourage the learner to complete a task or to study the course content more pleasantly. In recent years, the concept of gamification has fueled and excited the popular imagination. It has attracted the interest of academia as well as applied pedagogy. Its alleged motivating influence on students has made gamification a highly promising learning method. However, as its popularity grows, so does the corresponding controversy (Klabbers, 2018).

Although there is a satisfactory consensus regarding the definition of gamification, this is not the case regarding what constitutes a game mechanic or an element of a game or even game-based thinking.

The research is now turning toward the parts of games like storytelling, characters, challenge, immediate feedback, points, and achievements and trying to identify the most powerful elements that encourage learning ${ }^{1}$. A meta-analysis, which analyzed a number of potentially successful gaming variables, so as to take into account the widest possible range of gamification's conceptual heterogeneity (Sailer et al., 2017) showed that the inclusion of storytelling and the combination of competition and collaboration were the most effective factors. They help to promote the learning outcomes and changes in behavior, although their exact role remains somewhat unclear. It is also underlined the role of teacher identity as the cornerstone in shifting educational practices towards gamification.

\section{Escape Rooms as educational contexts}

Escape rooms are inspired by many kinds of games. They are action games based on a hypothetical scenario interspersed with riddles and their main components are challenge, competition, collaboration, exploration, experimentation, freedom of failure and feedback. The

[1] ${ }^{1} \mathrm{http}: / /$ karlkapp.com/2014-reflections-on-gamification-for-learning/ 
action begins with the confinement of players in a room where they are facing a difficult situation from which they must get out of, by solving puzzles or performing tasks according to the scenario. Theoretical background is to be found in motivation theory, self-determination theory and achievement goal theory (Sangkyun, Kibong, Lockee \& Burton, 2018: 39-42). The oldest example of an escape room was in Kyoto, Japan, and soon the kind proliferated all over the world (French \& Shaw, 2015) Since then, they have evolved into elaborate environments that offer exciting, enjoyable and constructive experiences for players of a wide range of demands, increasing their popularity.

In recent years, escape rooms have become one of the top leisure activities (Villar, 2018). They are defined as live-action group games where players discover clues, solve puzzles and work in one or more rooms to accomplish a goal in a limited time (Nicholson, 2015).

The philosophy and purpose of escape rooms in education is to design interactive experiences, not only to promote cognitive content but mainly to cultivate a positive attitude towards the educational process. Escape rooms are also beginning to receive attention as a potential practice to facilitate team-based research and ameliorate the performance of teams (Cohen, Griggs, Keebler, Lazzara, Doherty, Kanji, Gewertz, 2020).

Due to the novelty of the concept of the escape room, there is relatively limited literature on their use in education. The relative literature abounds in terms of their use in medicine \& Nicholson, 2017; Boysen-Osborn et al., 2018), pharmacy [Cain, 2019; Eukel et al., 2017; Cotner et al. 2018; Hermanns, 2018) physiotherapy and nursing in higher education but also in sciences, such as computer science (Borrego, 2017) chemistry (Dietrich, 2018) and the mathematics . (Ho, 2018; Glavas \& Stascik, 2017). In addition, there are initiatives aimed at promoting informal learning, such as earthquake preparation (Novak, 2019) or familiarizing students with the services of a campus (Wise, Lowe, Hill, Barnett \& Barton 2018; Walsh \& Spence, 2018).

In a recent exploratory study (Pan et al., 2017) which examined what opportunities escape rooms present as learning environments for improving collaboration and team dynamics, and how the design of escape rooms affects collaboration, it turned out that escape rooms provide people with opportunities to perform various nuanced collaborative acts and to practice varied social dynamics. These dynamics do not always match to social hierarchies and roles outside of the escape room. At other times, purely recreational rooms are being implemented in educational settings with the sole purpose of help participants acquiring soft skills such as teamwork, encouraging leadership and initiative, or harnessing collaboration and communication (Williams, 2018; Zhang, Lee, Rodriguez, Rudner, Chan \& Papanagnou, 2018), for the best distribution of tasks and time use.

Even more, over the last few years, also classroom digital escape rooms have been developed.

Escape rooms seem to overshadow other suggested educational activities for efficiently building a great communicative, collaborative and thriving team. The time pressure boosts effective communication, consensus building, and trusting relationships. They can also stand for a playful alternative to the standard worksheet or assessment.

\subsection{Design requirements}

When designing an original escape room, six key areas should be considered, as developed in previous projects of Arnab \& Clarke (2017), on the ground of Nicholson's founding white paper [37], based on a survey completed by proprietors of 175 escape room facilities around the world. Participants, Objectives, Topic, Riddles, Equipment and Evaluation. Each of these areas is further distinguished in specific parts, which are necessary components of the educational version of an escape room. Regarding the participants, the type of users and their needs must be 
taken into account as long as the difficulty level, the time available and the choice between cooperation or competition. Objectives can be focused on a subject, or be interdisciplinary, or even the emphasis can be on soft skills and problem-solving. The topic is developed on the canvas of a fictional narrative, autonomous or part of a broader one. These narratives cover a wide range of themes: horror (zombies, monsters) mystery (detective stories, crimes), adventure (Indiana Jones) rescue missions, espionage, diffusing a bomb, etc, and their main idea draws its origins from live-action role-playing games (LARP), such as Dungeons and Dragons.

The riddles ad the puzzles, which require a combination of knowledge, imagination, and skills to be solved, are given through instructions and hints. In general, the following conditions should be fulfilled: their seamless integration into the story, their ease of understanding, their ability to be solved based on the given information inside the room and their contribution to creating a suitable atmosphere in the room.

The preparation of the room entails its proper decoration, the selection of the necessary objects and props, the necessary technical support and the people who will frame it and make it plausible. The sense of urgency and the impending threat are usually maintained throughout the experience via various methods, such as time management, actors, settings, and effects, which seek to provoke emotional tension and mobilize the players to respond successfully in the predetermined time.

Finally, the evaluation of the educational implementation can be carried out through a questionnaire, observation, reflection, and feedback. As in other games, the concept of flow, in other words, of the state of total immersion in the process, is highly important (Csíkszentmihályi, 1996). Flow is the ideal mental and emotional state that a player can experience. When in flow condition, one is fully involved in a process and is absorbed exclusively by it. To achieve this in an escape room, one needs a balanced level of difficulty of the challenges. A too difficult challenge may lead to frustration and anxiety, while a too easy one to boredom and loss of interest. A well-designed game keeps players alert, avoiding both frustration and boredom (Clare, 2015).

The person supervising the room is known as the game master and it is up to him/her to help the players if they reach a dead end. The master of the game can be in the same room or monitor the progress of the players from elsewhere, via camera. He/she is responsible for introducing players to the game and saying goodbye to them at the end. During the game, he/she may need to give advice and extra hints.

\section{Save Odysseus: Escape Room Description}

The activity took place at the 2nd lower High School of Rhodes in the framework of extracurricular school activity and 10 five-member groups of students participated in it. It evolved into 4 different group meetings. The design was based on the philosophy of content gamification. Deviating slightly from the main narrative, we created a fictional narrative branch, which we used as the canvas for the development of our playful activities on the content. Upon completion, students were given questionnaires to assess their experience. The goal of the research was to record mainly the contribution of the escape room to cultivate collaboration and communication among the students, as decisive factors to enhance resilience in the school context, in other words, to empower them and prompt them to adopt a more positive attitude towards the learning process. The cognitive learning outcomes, having to do with the revision of certain key information regarding the Odyssey, which was included in the game, were measured by a typical test, but they are not included here. 
The main idea of the room was inspired by the Odyssey. At the fifth $(\varepsilon)$ rhapsody, Odysseus and his companions arrive in Faiakia (Sheria), the island of Faiakes. During the feast, offered by the Faiakes for Odysseus, he started narrating his adventures over the past ten years. At first, they were excited to listen to him, but at some point, Alcinous, the king, felt offended, because he thought that all these stories were fictional and fabricated and that his guest was an impudent imposter (this narrative twist was invented for the sake of the plot and despite the originally narrated events in the epic). The next day, when Odysseus' companions woke up, they searched for him in vain. He had disappeared. Suddenly, they were dragged by force into a dungeon, ignoring why. So, they were first and foremost called upon to find out why they had been brought there, by deciphering a secret coded message that Odysseus had managed to send them through a newspaper clipping of the time "Figure 2", before he was imprisoned. This is the entry point of the game.

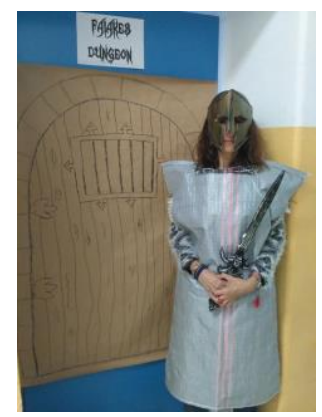

Figure 1. The master of the game as the Dungeon's Guard.

The players, as Odysseus' companions, have to make decisions. Which front page is the right one, how will they decipher the message? They must solve a riddle to find the deciphering code and then apply it. When done, they know that they must prove the truth of Odysseus' narration, in order to be set free and continue their travel back to Ithaca. To create the right atmosphere, we used sacks as uniforms for the convicts, dark papers and curtains to darken the room and a creepy music carpet to create scary atmosphere.

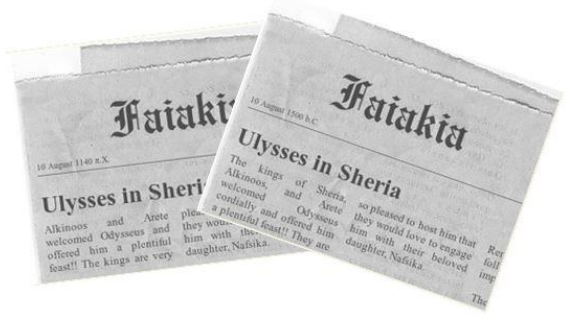

Figure 2. The newspaper clippings

The "master of the game" with a tunic, sword, and helmet, contributed to the more convincing reception of the circumstances. "Fig. 1". Throughout the game, a candle was displayed on the wall that lingered until the 20 -minute deadline, given to players to complete the tests.

The contents of the room were:

1. A map of the Mediterranean sea (in order to spot the places of Odysseus' journey)

2. A special torch to be used to reveal notes written by invisible ink

3. A ball of twine

4. A mirror

5. A blackboard or a long rectangular piece of paper

6. Papers and pens (for the students to keep notes and make calculations)

7. Cards with names of persons that appear in the Odyssey (printable resources) 
8. Red-reveal cards with hidden numbers (printable resources)

9. $3 \mathrm{~d}$ paper glasses (blue and red)

10. The pieces of 3 jigsaw puzzles (hand made)

11. A pc with a projector (for the countdown clock- candle-like)

12. A tablet (for the purpose game designed as the last step)

13. A box/bag (to keep the door key inside)

14. A padlock

The room was designed on a sequential model, including a linear sequence of 6 puzzles (5 missions), that required solving the mystery, finding the clues, unraveling the riddles, gathering numbers, putting them in right order and finally cracking the code of the padlock, that kept them imprisoned in the dungeon.

\subsection{The missions}

1st mission: The classroom whiteboard or a rectangular large sheet of paper on the wall lists puzzles that describe persons involved in the Odyssey (e.g. the princess in an unresponsive love equals Nafsika, Alcinous daughter). In various places on the surface, color stickers have been pasted. Players must connect according to the instructions, every two points of the same color between them, using the ball of twine in the room. At the points where specific lines intersect, some puzzles are to be found. They have to solve the riddles and then locate among a pile of cards with names of persons related to the Odyssey, the ones that correspond to the descriptions of the puzzles. In the end, they correspond the first letter of each name to its number in Alphabet. Finally, they add the numbers together and have the first digit, to unlock the padlock. For the 2nd mission, the players have the following instructions: The appearances are deceiving, according to a famous proverb. Perhaps the Cyclops, though single-eyed, could see better. Find a secret number associated with the Odyssey.

Then make the jigsaw puzzles. One of them refers to an adventure of Odysseus. Locate its place on the map by "shedding "light in the darkness of ignorance". Subtract the number you find (and refers to the order in which Odysseus and you visited this place) from the number you found earlier and finally sum up all the digits until you reach a single digit.

The first riddle can be solved through some cards made with the red reveal technique, where various numbers are hidden in a colorful mosaic "Fig. 3". With the help of the pair of 3d glasses, closing the eye with the blue color glass and seeing only through the red glass, they will be able to distinguish them. They have to choose the number 41, which is the number of days that the narrative of the Odyssey takes place.

Then they have to make three jigsaw puzzles with fruits and recognize the lotus, which refers to the homonymous adventure of Odysseus (at the Lotus-eaters). Finally, they have to look for hidden information on the map of the Mediterranean sea. Various stops of Odysseus' voyage have been marked with invisible ink there. To locate them, they are called upon to shed "light on the darkness of ignorance," that is, to use the special torch that can reveal the invisible writing. After completing all the steps, they come out with the second valuable digit. 


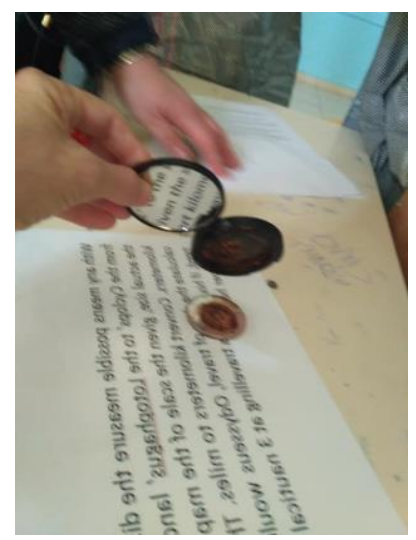

Figure 3. The reverse message

3rd mission: The players find a reverse message "Fig. 2". They can read it with the help of an object in the room (the mirror). Then, they have to follow the instructions. The message asks them to measure the distance between the land of Lotus-eaters and the land of Cyclops, with whatever possible means (there is no ruler or meter, so they must use the twine) and find the real proportions by taking into account the map's dimensions noted in the lower part of it. Then, they have to transform the kilometers to nautical miles and calculate the days Odysseus needed to move from one place to the other, if the boat's average speed had been 3 miles per hour. This is the third digit to salvation.

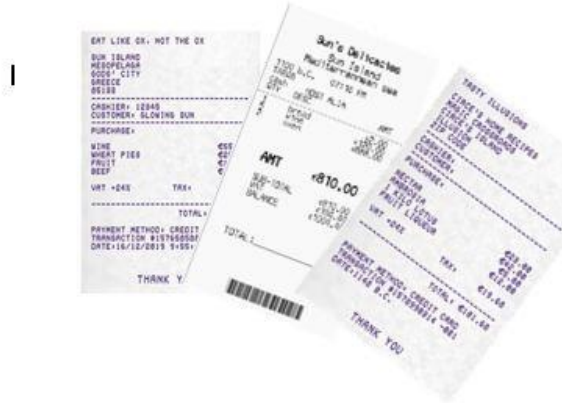

Figure 4. The restaurants' receipts

4th Mission: Players find fake receipts from the "restaurants" Odysseus and his companions visited during their journey, among which only one is right. To locate it, they must observe the date and the products they consumed. Specifically, the correct one is from the island of the Sun, around 1100 BC. where it states that they also ate beef (which was prohibited and caused Sun's anger and their expulsion from his island). Having added all the numbers in the bill, until they reach a single digit, they have the next number for the padlock's code. Right after, they must decrypt a message written in Pigpen code and follow its instructions. Specifically, they are asked to solve a labyrinth puzzle with letters, in which the exit route forms the sentence "place the numbers in ascending order".

Before they are allowed to use the 4 digit code, there is a final verification trial.

5th mission: In an informative text about the Odyssey, they have to fill in the missing letters in several words and form a phrase out of them, which is: "Play the game on the tablet". https://www.purposegames.com/game/iJFYh60zEtt

There, they will have to successfully play a specially created game about Odysseus' journey. They are called to recognize on an interactive map. Some places already visited by them during their journey. For extra help, they can return to the map with the hidden information in invisible ink. 


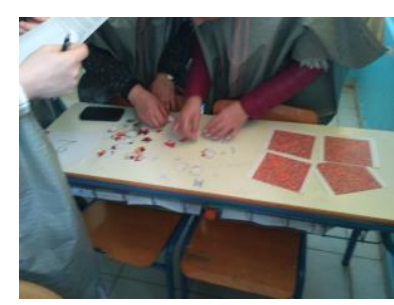

Figure 5. Red reveal cards and jigsaw puzzles

\subsection{Evaluation}

The key research question was whether participating in an escape room enhances collaboration and contributes to mental resilience in the school environment especially for vulnerable (with low performance) students. Our null hypothesis was that there is no correlation between participation in the escape room and the desired results, which we seek to achieve. Hence, we examined the relationship between the level of participation and collaboration and the positive emotions generated with the desire to repeat a similar activity and the general perception of the school environment. In addition, with the questionnaire, we meant to measure which coefficients were activated during the participation in the room, and which functioned as a positive or negative experience, in order to get feedback at the design level, for future use.

The questionnaire comprised of the following questions (keywords in the brackets):

Gender: boy/girl

General passing grade from previous school year:

Have you ever participated in an escape room before?

Did you collaborate with your team? (collaboration)

Did you take leadership initiative?(leadership)

Did you follow the suggestions of others? (compliance)

Did you work individually?(individuality)

Were you energetic and involved in the action? (engagement)

Were you passive - or indifferent?(passivity)

Did you encourage the efforts of others? (supportive encouragement)

Were you excited about solving the puzzles? (enthusiasm)

Were the puzzles easy?(ease)

Did the time pressure make the action more interesting? (time-pressure)

Did you like the idea of competing with the other teams? (competition)

Were you anxious to solve the puzzles? (anxiety)

Was stress reduced by participation in the group? (teamwork- stress mitigation)

Did solving puzzles required creative-divergent thinking? / (creativity)

Did you have fun? (fun)

Would you like similar activities to take place at school? (desire of repetition)

Do you think such activities have educational value?(educational value)

Do such actions make school more enjoyable and friendly? (resilience).

The results were tested in the SPSS statistical tool. Initially, a reliability test (Cronbach's Alpha) of the sample was performed and the result showed a strong degree of reliability (.730). In the normality test (we chose Shapiro-Wilk, because the size of the sample was exactly 50 ) then performed, the sample showed irregular distribution $(p<0.5)$, so that non-parametric tests were applied.

A first check of the mean values of the variables gave high scores, approaching "agree"(4 out of 5) and even surpassing it, on the following axes: teamwork- stress mitigation (3.74), love for competition (3.78), enthusiasm (3.78), supportive encouragement (3.82), desire of repetition of 
the activity (3.86), fun (3.88), collaboration (3.90), creativity (3.96), school resilience (4.10), educational value (4.12) active engagement (4.24). The high score in the question, if such activities make school more enjoyable and friendly combined with the rest scores, argues in favor of the hypothesis, that an educational escape room can reinforce the positive attitude towards school itself, in other words, it can support resilience through fun and collaboration.

Checking the effect of gender on the above variables, by the Mann-Whitney non-parametric test, a statistically significant difference was found between boys and girls, with boys giving more positive replies (agree to strongly agree) as follows:

Collaboration, $\mathrm{U}=154,500, \mathrm{p}=0.01$, encouragement, $\mathrm{U}=198,000 \mathrm{p}=0.021$, enthusiasm, $\mathrm{u}=87,000$, $\mathrm{p}=0.001$, creativity, $\mathrm{U}=208,000, \mathrm{p}=0.022$, competition, $\mathrm{U}=28,500, \mathrm{p}=0.032$, educational value, $\mathrm{U}=182,000, \mathrm{p}=0.008$

Then, the effect of students' school performance on the variables was checked, by the MannWhitney non-parametric test, as well. First, we recoded the grades' scale into two new variables, the one including the grades ranging between almost well and well (10-15) and the other between very well and excellent (16-20). Statistically significant differences were observed as follows:

In all cases, those whose school performance was lower, between almost well to well (10 to 15) outscored the other group in positive replies in: collaboration $(\mathrm{U}=90,500, \mathrm{p}=0.001)$, encouragement $(U=157,000, p=0.048)$, enthusiasm $(U=127,500, p=0.006)$, anxiety $(U=23,500$, $\mathrm{p}=0.001)$, teamwork- mitigation of anxiety $(\mathrm{U}=36,500, \mathrm{p}=0.001)$, desire of repetition of the activity $(150,000, \mathrm{p}=0.031)$, resilience $(\mathrm{U}=107,500, \mathrm{p}=0,002)$

Afterward, taken as independent variable one's previous participation in an escape room, statistically, significant differences were observed in the following dependent variables, as follows: Those who had a previous experience replied more positively in the competition factor $(u=205,000, p=0.049)$, the creativity $(U=166,500, p=0.003)$ and the desire for repetition $(\mathrm{U}=152,500, \mathrm{op}=0.002)$. Those who did not have a previous experience were more enthusiastic about their participation $(\mathrm{U}=196,500, \mathrm{p}=0.03)$.

Subsequently, we compared some core variables to our research question using the Wilcoxon signed-ranks test. We found out that between individual and collaborative work, the second one outweighs significantly (47/50 declared that collaborated than acting individually) $(\mathrm{Z}=-5796, \mathrm{p}=$ $0.001)$.

Respectively comparing the questions:

Were you passive - or indifferent? / Were you energetic and involved in the action? 45/50 declared themselves energetic than passive $(Z=-6,091, p=0.001)$.

Was stress reduced by participation in the group? 22/50 said that teamwork alleviated their anxiety and $17 / 50$ gave a neutral reply $(Z=-1994, p=0.046)$.

Finally, we searched for correlations between variables, after checking that our data met the necessary requirements. The scatter plot between the variables checked, was examined and there did not exist nonlinear indications or significantly deviating values.

So, between the variables: "Do such actions make school more enjoyable and friendly?" and "Would you like similar activities to take place at school?" a moderate correlation was found (,544), $\mathrm{p}=0.001$.

Between the variables: "Did you collaborate with your team?" and "Do such activities make school more friendly and enjoyable?", a low (to moderate) correlation was found $(, 417) \mathrm{p}=0.003$. Between the variables: "Did you collaborate with your team?" and "were you excited about solving the puzzles?" a strong correlation was found $(, 680), \mathrm{p}=0001$. 
Between the variables: "Did you collaborate with your team?" and "Would you like similar activities to take place at school?" a low correlation was found $(, 310), \mathrm{p}=0.029$.

\section{Conclusions}

The research outcomes supported our initial belief that an escape room as a content gamification activity in education enhances collaboration and mental resilience at school, proving the null hypothesis invalid (Null Hypothesis: The participation in the escape room has no correlation with school resilience and it is not a pedagogically beneficial practice). In regard with the research questions, the escape room proved to have a high collaboration value (qu. 1) and even more, among low-performance, vulnerable students. The same with active engagement (qu. 2), mitigation of anxiety through team-work (qu. 3), fun (qu. 4), desire for repetition (qu. 5) and pedagogical significance educational value (qu. 6). Students' satisfaction was found dependant on previous participation in escape rooms and gender (boys were more enthusiastic) (qu. 7). Previous experience was proved as a supporting factor for the competitive spirit, desire for further participation and creative thinking.

The independent variable "gender" showed that boys enjoyed the activity more than girls, collaborated more enthusiastically, preferred competition with other teams and activated their creativity to respond to the missions. As far as it concerns the last and most important question, vulnerable students were benefitted from the experience more than those with high performance and expressed more fervently their desire to participate in similar activities in the future, which to their opinion- makes school more friendly. This statement is interpreted as a factor of school resilience. Comparing variables we found out that collaboration outweighed individuality, engagement outweighed passivity, and teamwork functioned as a beneficial factor against anxiety. Even more, the correlation test (bvariate) showed -among other less significant results- a strong correlation between collaboration and excitement (0.680) and a moderate one (0.544) between the desire for further participation in similar activities with the opinion that such activities make school more friendly (the factor that we had named resilience).

\subsection{Discussion- restrictions}

Taking into account the limitations of our study, due to its restricted size of participants $(N=50)$, it is self-evident that a larger scale study would increase our outcomes' reliability. Limitations of the generalizability and the reliability of our outcomes also emanate from the non-random choice of our participants, which did not constitute a representative sample of the general school population of their age. More similar activities should be implemented, including various school subjects or interdisciplinary and addressing different school levels, to contribute to a more spherical perception of the contribution of the escape rooms in education, concerning either cognitive or pedagogical goals. Vulnerability of school population could as well be measured with different variables, others than low performance, while school resilience could be also conceived in a more broad and comprehensive way, including various factors, others than positive attitude towards learning and active participation.

\section{References}

Anderson, C. A., \& Dill, K. E. (2000). Video Games and Aggressive Thoughts, Feelings and Behavior in the Laboratory and in Life. Journal of Personality and Social Psychology, (78)4.

Anderson, R. (2008). Implications of the information and knowledge society for education. In J. Voogt, \& G. Knezek, (Eds.). International handbook of information technology in primary and secondary education (pp. 5-22). New York: Springer.

Arnab, S., \& Clarke, S. (2017). Towards a transdisciplinary methodology for a game-based intervention development process. British journal of educational technology, 48(2), 279-312.

Baker, A., Navarro, E. O., \& Van Der Hoek, A. (2005). An experimental card game for teaching software engineering processes," J. Syst. Software, 75(1), 3-16. https://doi.org/10.1016/j.jss.2004.02.033 
Becker K., \& Nicholson S. (2016). Gamification in the Classroom: Old Wine in New Badges. In K. Schrier (Eds.) Learning Education and Games. Volume Two: Bringing Games into Educational Contexts (pp. 61-86). ETC Press.

Bernard, B. (1991). Fostering Resiliency in Kids: Protective Factors in the Family, school, and Community. San Francisco, CA: WestEd Regional Educational Laboratory.

Binkley, M., Erstad, O., Herman, J., Raizen, S., Ripley, M., \& Rumble, M. (2012). Defining Twenty-First Century Skills. In Griffin, P., McGaw, B. \& Care, E. (Eds.). The Assessment and Teaching of 21st Century Skills. Dordrecht: Springer.

Bogost, I. (2011). Gamification is Bullshit. Retrieved from http://bogost.com/blog/gamification_is_bullshit/

Borrego, C., Fernández, C., Blanes, I., \& Robles, S. (2017). Room escape at class: Escape games activities to facilitate the motivation and learning in computer science. J. Technol. Sci. Educ., 7(2), 162-171.

Boysen, M., Osborn, S., Paradise, \& Suchard, J. (2018). The toxiscape hunt: An escape room-scavenger hunt for toxicology education. J. Educ. Teach. Emerg. Med., 3(1), 9-19.

Bruckman, A. (1999). Can educational be fun? Paper presented at the Game Developers Conference '99, San Jose, CA.

Cain, J. (2019). Exploratory implementation of a blended format escape room in a large enrollment pharmacy management class, Currents PharmacyTeach. Learn., Jan., 11(1), 44-50.

Caldwell, B., \& Longmuir, F. (2010). Curriculum and Pedagogy for the 21st Century Challenges for School Leaders. In Proceedings of the Annual Conference of the Queensland Studies Authority (QSA) on the theme of Shared Vision: An Australian Curriculum P-12, 29 April 2010, Brisbane Convention and Exhibition Centre.

Clare, A. (2015). Flow. In Escape the Game: How to Make Puzzle and Escape Rooms. (1st ed). Toronto: Wero Creative.

Cohen T. N., Griggs A. C., Keebler J. R., Lazzara E. H., Doherty S. M., Kanji A. F., Gewertz B. L., (2020). Using Escape Rooms for Conducting Team Research: Understanding Development, Considerations, and Challenges, Simulation \& Gaming.

Cotner, S. K., Smith, M., Simpson, L., Burgess, D. S., \& Cain J. (2018). Incorporating an "escape room" game design in infectious diseases instruction. Open Forum Infect. Dis., 5.

Csíkszentmihályi, M. (1996). Creativity: Flow and the Psychology of Discovery and Invention. New York: Harpeerennial.

Dempsey, J., Haynes, L., Lucassen, B., \& Casey, M. (2002). Forty simple computer games and what they could mean to educators. Simulation \& Gaming, 33(2), 157-168.

Deterding, S., Dixon, D., Khaled, R., \& Nacke, L. (2011). From game design elements to gamefulness: defining "gamification". In A. Lugmayr (Ed.) Proceedings of the 15th International Academic Mindtrek Conference: Envisioning Future Media Environments(pp. 9-15). New York: ACM. https://doi.org/10.1145/2181037.2181040.

Dietrich N. (2018). Escape classroom: The leblanc process. An educational `Escape Game'. J. Chem. Educ, 95(6), 996-999.

Dillenbourg, P., Baker, M. J., Blaye, A., \& O’Malley, C. (1995). The evolution of research on collaborative learning. In E. Spada \& P. Reiman (Eds.), Learning in bumans and machine: Towards an interdisciplinary learning science (pp. 189-211). Oxford, UK: Elsevier.

Eukel H. N., Frenzel J. E., \& Cernusca, D. (2017). Educational gaming for pharmacy students. Design and evaluation of a diabetes-themed escape room. Amer. J. Pharmaceutical Educ., Sep., 81(7), 6265.

Fadel, C., \& Trilling, B. (2012). Twenty-first century skills and competencies. In N. M. Seel (Ed.), Encyclopedia of the sciences of learning (pp. 3353- 3356). New York: Springer.

French, S., \& Shaw, J. M. (2015). The unbelievably lucrative business of escape rooms. Retrieved https://www.marketwatch.com/story/the-weird-new-world-of-escape-roombusinesses-2015-07-20.

Hermanns, M. B., Deal, S., Hillhouse, J. B., Opella, C., Faigle, I. V., Campbell, \& Robert, H. 2018. Using an 'Escape Room' toolbox approach to enhance pharmacology education. J. Nurs. Educ. Pract., 8(4), 89-95. 
Higgins, G. O. (1994). Resilient adults: Overcoming a cruel past. Jossey-Bass.

Ho, A. M. (2018). Unlocking ideas: Using escape room puzzles in a cryptography classroom.' PRIMUS, 28(9), 835-847.

Glavas, A., \& Stascik, A. (2017). Enhancing positive attitude towards mathematics through introducing Escape Room games. Mathematics education as a science and a profession, 281-293.

Huizinga, J. (1944). Homo Ludens; A Study Of The Play-Element In Culture. London: Routledge \& Kegan Paul.

Jenkins, H., Purushotma, R., Weigel, M., Clinton, K., \& Robison, A. J. (2009). Confronting the challenges of participatory culture: Media education for the 21st century. Mit Press. Retrieved from https://www.macfound.org/media/article_pdfs/JENKINS_WHITE_PAPER.PDF

Klabbers, J. H. (2018). On the architecture of game science. Simulation \& Gaming, 49(3), 207245. https://doi.org/10.1177/1046878118762534.

Kuhn, D. (2015). Thinking together and alone. Educational Researcher, 44, 46-53.

Landers, R. N., Auer, E. M., Collmus, A. B., \& Armstrong, M. B. (2018). Gamification science, its history and future: definitions and a research agenda. Simulation \& Gaming, 49(3), 315337. https://doi.org/10.1177/1046878118774385.Return

Lerner, R. M., Almerigi, J. B., Theokas, C., \& Lerner, J. V. (2005). Positive youth development: A view of the issues. The Journal of Early Adolescence, 25, 10- 16.

McClarty, K. L., Orr, A., Frey, P. M., Dolan, R. P., Vassileva, V., \& McVay, A. (2012). A literature review of gaming in education. Gaming in education, 1-35. Mitchell, A. \& Saville-Smith, C. (2004). The use of computer and video games for learning. A review of the literature. London: The Learning and Skills Development Agency.

Monaghan S. R., \& S. Nicholson. (2017). Bringing escape room concepts to pathophysiology case studies. HAPS Educ., Jul. 21 (2), 49-65.

Moula, E. (2012). Is edutainment just a chocolate-coated broccoli? Computer games and history: intersections, interactions, learning advantages and strategies. In Proceedings of International Scientific

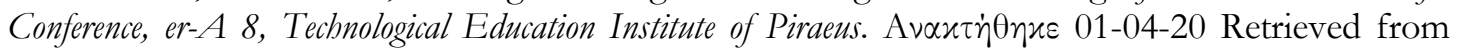
http://www.pee.gr/wp-content/uploads/eRA8_1-481.pdf

Nicholson, S. (2015). Peeking behind the locked door: A survey of escape room facilities. Retrieved from http://scottnicholson.com/pubs/erfacwhite.pdf

Nicotera, N., \& Laser-Maira, J. A. (2016). Innovative Skills to Support Well-being and Resiliency in Youth. Oxford University Press.

Novak, J., Lozos, J. C., \& Spear, S. E. (2019). Development of an interactive escape room intervention to educate college students about earthquake preparedness. Natural Hazards Review, 20(1).

Papadakis, S., \& Kalogiannakis, M. (2019). Evaluating the effectiveness of a game-based learning approach in modifying students' behavioural outcomes and competence, in an introductory programming course. A case study in Greece. International Journal of Teaching and Case Studies, 10(3), 235-250.

Prensky, M. (2001). Digital Natives, Digital Immigrants. On the Horizon. MCB University Press, 9(5).

Sailer, M., Hense, J. U., Mayr, S. K., \& Mandl, H. (2017). How gamification motivates: an experimental study of the effects of specific game design elements on psychological need satisfaction. Computers in Human Behavior, 69, 371380. https://doi.org/10.1016/j.chb.2016.12.033.

Sangkyun, K., Kibong, S., Lockee, B. \& Burton, J. (2018). Gamification in Learning and education. Enjoy Learning Like Gaming. Springer.

Squire K. D. (2006). From Content to Context: Videogames as designed experience. Educational Researcher, 35(8), 19-29.

Squire, K., \& Jenkins, H. (2004). Harnessing the power of games in education'. Insight (3)1, 5-33.

Steinkuhler, C., Squire, S. \& Barab, S. (2012) (Eds) Games, Learning, and Society: Learning and Meaning in the Digital Age. Cambridge: Cambridge University Press.

Strickland, H. P., \& Kaylor, S. K. (2016). Bringing your a-game: Educational gaming for student success. Nurse Education Today, 40, 101-103. https://doi.org/10.1016/jnedt.2016.02.014 
Tang, S., \& Hanneghan, M. (2011). State-of-the-art model driven game development: A survey of technological solutions for game-based learning. Journal of Interactive Learning Research, 22(4), 551605.

Villar A. (2018). Ocio y turismo millennial: El fenómeno de las salas de escape. Cuadernos Turismo, 41, 615636.

Voogt, J., Roblin, P. N. (2010). 21st Century Skills. Discussion paper. Retrieved from http://archief.kennisnet.nl/fileadmin/contentelementen/kennisnet/Bestanden_Feddo/21stCentury-Skills.pdf

Wagner, T. (2010). The global achievement gap: Why even our best schools don't teach the new survival skills our children need — and what we can do about it. New York: Basic Books.

Walsh, B., \& Spence, M.(2018). Leveraging escape room popularity to provide 1rst-year students with an introduction to engineering information. In Proc. Can. Eng. Educ. Assoc. (CEEA) Conf., Dec. 2018, 1-9.

Williams P. (2018). Using escape room-like puzzles to teach undergraduate students effective and efficient group process skills, In Proc. IEEE Integr. STEM Educ. Conf., Mar. 2018, 254-57

Wise H., Lowe J., Hill, A., Barnett, L., \& Barton, C. (2018). Escape the welcome cliché: Designing educational escape rooms to enhance students' learning experience J. Inf. Literacy, 12(1), 86-96.

Zhang, X. C., Lee, H., Rodriguez, C., Rudner, J., Chan, T. M., \& Papanagnou, D. (2018). Trapped as a group, escape as a team: applying gamification to incorporate team-building skills through an 'escape room' experience. Cureus, 10(3). 